\title{
HCV Triple Therapy is Equally effective in African-Americans and Non-African-Americans
}

\author{
John Wysocki • Celeste Newby • Luis Balart • \\ Nathan Shores
}

Received: 2 April 2014 /Revised: 9 July 2014 / Accepted: 23 July 2014 / Published online: 15 August 2014

(C) W. Montague Cobb-NMA Health Institute 2014

\begin{abstract}
Real-world experience with HCV medications differs from original the index studies. AfricanAmericans (AA) are commonly underrepresented in clinical trials. Therefore, we hypothesized that postmarketing experience with the first generation HCV protease inhibitors (PI) (telaprevir and boceprevir) differs from premarket reports in clinically meaningful ways regarding patient race. We conducted a single-center, retrospective, observational analysis to evaluate the efficacy of $\mathrm{HCV}$ triple therapy in a nontrial setting. Clinical response and quantitative laboratory values were compared between AA and non-AA patients throughout the duration of treatment. Patients were included if they started triple therapy treatment after July 2011 and are not a part of any other clinical/pharmaceutical trials. Thirty-five patients with $\mathrm{HCV}$ genotype 1 were included in this study -22 non$\mathrm{AA}$ and 13 AAs. AAs had a higher BMI compared to non-AA (33.6 vs 28.8, $p=0.046$ ). "High" baseline HCV RNA values (above 800,000 copies) were more prevalent in AA (69.2 \%) vs non-AA (63.6\%). AA had less favorable IL28B genotypes; only $7.7 \%$ had type CC compared to $40.9 \%$ of non-AA. SVR12 was achieved $82 \%$ of the time with each PI but there was no significance in type of PI used $(p=1.00)$. Patients with cirrhosis were less likely to achieve SVR12 (40\%) compared to those without cirrhosis $(88 \%, p=0.013)$. Thirty-three percent of the patients who received blood transfusions did not achieve SVR12 while every patient who did not require
\end{abstract}

Electronic supplementary material The online version of this article (doi:10.1007/s40615-014-0039-x) contains supplementary material, which is available to authorized users.

J. Wysocki $(\bowtie) \cdot$ C. Newby $\cdot$ L. Balart $\cdot$ N. Shores

Department of Gastroenterology and Hepatology, Tulane University

School of Medicine, 1430 Tulane Avenue, New Orleans, LA 70112, USA

e-mail: jwysocki@tulane.edu transfusion achieved SVR12 $(p=0.001)$. All patients with IL28B genotype CC achieved SVR12 ( $p=0.007)$. SVR12 did not differ among AA (77 \%) and non-AA (86.4 \%) $(p=0.528)$.

Keywords Hepatitis C · Hepatitis treatment · Triple therapy · Real-world experience $\cdot \mathrm{HCV}$ treatment

\section{Introduction}

The prevalence of hepatitis $\mathrm{C}$ is higher in African-Americans compared to other races in the USA. According to NHANES III data, African-Americans had a prevalence of $3.2 \%$ in the reported population compared to $1.5 \%$ in the general US population [1-3]. Historically, African-Americans have been underrepresented in HCV trials. Mehlia et al. [4] concluded that African-Americans were $65 \%$ less likely to be eligible for treatment given comorbidities such as cytopenia, diabetes mellitus, and chronic kidney disease.

Given the prevalence of disease, especially in AfricanAmerican men born between 1945 and 1965, HCV related complications such as cirrhosis, decompensation, and HCC are a growing health concern [5]. A study from the National Center for Health Statistics suggests that racial disparities exist in HCV infection, with Africa-Americans having a twofold higher rate of death than expected [6]. Such trends are expected to continue, with the number of HCV cases and decompensation events peaking between 2020 and 2022.

Enrollment of African-Americans in therapeutic trials for HCV has been disproportionate. According to 2011, US Census data estimated that $13.6 \%$ of the population was African-American. The most recent major trials analyzing triple therapy treatment in $\mathrm{HCV}$ were able to enroll 7-27\% of the study participants as African-Americans [7-14]. Although improvements have been made in recruitment 
diversity, the reality is that these are sponsored trials, with inclusion and exclusion criteria. A large percentage of African-Americans have not been eligible. There are no studies that describe this population of patients who do not qualify or do not choose to participate in drug trials. Furthermore, no single center has published real-world experiences that clinicians may expect to see from their diverse set of patients undergoing triple therapy for HCV. The goal of this study was to offer the largest cohort of triple therapy patients, with a specific focus on comparing the treatment experiences of Africa-Americans to non-African-Americans.

\section{Methods}

\section{Study Design}

This approved study is a retrospective, observational design conducted in a single center (Tulane Medical Center, Hepatology Clinic, New Orleans, LA, USA). Eligible candidates were patients with chronic HCV, genotype 1 as identified by detectable levels of serum HCV RNA (Roche COBAS TaqMan HCV assay), and HCV Genotype analysis (LabCorp, Burlington NC). IL28B genotype polymorphism (RT-PCR) was determined by a serum sample (LabCorp, Burlington $\mathrm{NC}$ ). Severity of fibrosis was determined by liver biopsy, (FibroSure LabCorp, Burlington NC), or radiographic evidence. Additionally, eligibility required that subjects initiate and complete HCV triple therapy after July 2011, could not be a part of any other clinical/pharmaceutical trials, receive at least one dose of HCV protease inhibitor, and be at least 17 years of age. Patients were excluded if they received triple therapy treatment as part of an industry-sponsored clinical trial. Also, patients could not have concurrent HIV infection, hepatocellular carcinoma. Individual treatment regimens were based on the clinical judgment of his/her treating hepatologist. Data including labs, medications, clinic notes, nursing visits, and hospitalization records were extracted by hand from individual patient charts.

\section{End Points}

The primary end points were the comparative SVR 12 of African-Americans versus White patients. Secondary endpoints included medication dosing, side effects, transfusion requirements, BMI, gender, previous treatment outcome, genotype, IL28B polymorphism, and final outcome (SVR12). Chen et al. recently demonstrated that the positive predictive value of SVR 12 for SVR 24 was $98 \%$ and negative predictive value was $99 \%$ [15]. As SVR12 was a treatment endpoint for this study, final treatment outcomes could vary slightly.
Statistical Analysis

Descriptive statistics for patients were reported in Table 1. A chi-squared test was used to compare categorical variables between White Americans and African-Americans. Continuous variables were summarized as means with standard deviation. ANOVA was performed to compare treatment outcomes relative to SVR12, ethnicity, and protease inhibitor. Significance was chosen to be $<0.05$. All statistical analysis was performed using SPSS.

\section{Results}

A total of 35 patients were included in this study -22 White Americans and 13 African-Americans. Demographic details were compared between African-Americans and White Americans and are shown in Table 1. Significant differences between the two groups included BMI, baseline HCV RNA, IL28B polymorphism, history/response to previous HCV treatment, and platelet count at baseline. African-Americans were more likely to have a higher BMI compared to White Americans (33.6 vs 28.8, $p=0.046$ ). Baseline HCV RNA values considered high (above 800,000 copies) were more prevalent in African-Americans (69.2\%) vs White Americans (63.6\%). African-Americans had less favorable IL28B genotypes; only $7.7 \%$ had type CC compared to $40.9 \%$ of White Americans. African-Americans were more likely to be treatment naïve (76.9\%) compared to White Americans (40.9 \%). In six cases, IL28B was not collected and was therefore unknown. Finally, the baseline blood counts, specifically platelet count, were significantly higher prior to treatment initiation in African-Americans $(224,000)$ compared to White Americans $(177,000)$.

Table 2 shows which variables were significantly associated with virus negativity 12 weeks after completion of treatment (SVR12). Patients with cirrhosis were less likely to achieve SVR12 (50 \%) compared to those without cirrhosis $(87 \%, p=0.013)$. Approximately $33 \%$ of the patients who received blood transfusions did not achieve SVR12 yet $100 \%$ of the patients who achieved SVR12 did not require blood transfusion $(p=0.001)$. All patients with IL28B genotype CC achieved SVR 12 compared to only $40 \%$ with Type TT ( $p=$ 0.007). While blood transfusion was significant for SVR12, neither ribavirin dose reduction $(p=0.384)$ nor erythropoietin injection ( $p=0.686$ ) requirement were found to be significant. Despite these observations, race (African-Americans-37\% of study population, White Americans - 63\% of study population) was not found to be significant $(p=0.528)$ in this study in predicting SVR12. Thirty-three percent of non-SVR patients and $25 \%$ of SVR patients were hospitalized, most commonly for weakness (Supplemental Table 2). 
Table 1 Selected baseline characteristics by race
${ }^{\text {a }}$ Race was self-reported. No Hispanic, Latino, or Asian patients were included in this consecutive patient analysis

${ }^{\mathrm{b}}$ BMI was according to height and weight at initiation of triple therapy

${ }^{\mathrm{c}}$ Cirrhosis was determined according to radiographic, histologic, or serologic criteria

\begin{tabular}{|c|c|c|c|}
\hline & White American $(n=22)^{\mathrm{a}}$ & African-American $(n=13)^{\mathrm{a}}$ & Significance \\
\hline Age (SD) & $49.3(12.1)$ & $49.9(10.0)$ & 0.887 \\
\hline $\mathrm{BMI}(\mathrm{SD})^{\mathrm{b}}$ & $28.8(5.2)$ & $33.6(8.6)$ & 0.046 \\
\hline Male sex $(\%)$ & $12(54.5)$ & $4(30.8)$ & 0.183 \\
\hline Married (\%) & $11(50)$ & $7(53.9)$ & 0.832 \\
\hline Job $(\%)$ & $15(68.2)$ & $6(46.2)$ & 0.210 \\
\hline Insurance (\%) & & & 0.125 \\
\hline Private & $18(81.8)$ & $8(61.5)$ & \\
\hline Medicare or Medicaid & $4(18.2)$ & $4(30.8)$ & \\
\hline Uninsured & $0(0)$ & $1(7.7)$ & \\
\hline HCV RNA baseline $>800,000(\%)$ & $14(63.6)$ & $9(69.2)$ & 0.040 \\
\hline Genotype & & & 0.772 \\
\hline 1a $(\%)$ & $19(86.2)$ & $10(76.9)$ & \\
\hline $1 \mathrm{a} / 1 \mathrm{~b}(\%)$ & $1(4.5)$ & $0(0)$ & \\
\hline $1 \mathrm{~b}(\%)$ & $2(9.1)$ & $3(23.1)$ & \\
\hline IL28B & & & 0.008 \\
\hline $\mathrm{CC}(\%)$ & $9(40.9)$ & $1(7.7)$ & \\
\hline CT $(\%)$ & $10(45.5)$ & $4(30.8)$ & \\
\hline TT $(\%)$ & $1(4.5)$ & $4(30.8)$ & \\
\hline Unknown (\%) & $2(9.1)$ & $4(30.8)$ & \\
\hline Previous HCV treatment & & & 0.022 \\
\hline Stopped due to side effects (\%) & $2(9.1)$ & $0(0)$ & \\
\hline Naïve (\%) & $9(40.9)$ & $10(76.9)$ & \\
\hline Nonresponder (\%) & $4(18.2)$ & $2(15.4)$ & \\
\hline Relapse (\%) & $4(18.2)$ & $1(7.7)$ & \\
\hline \multicolumn{4}{|l|}{ Platelet count (baseline) } \\
\hline Mean baseline - $100 \mathrm{~K}$ (SD) & $177(61)$ & $224(67)$ & 0.045 \\
\hline 100 to $150 \mathrm{~K}(\mathrm{SD})$ & $8(36.4)$ & $2(15.4)$ & \\
\hline$>150 \mathrm{~K}(\mathrm{SD})$ & $14(63.6)$ & $11(84.6)$ & \\
\hline Cirrhosis $^{\mathrm{c}}$ & & & 0.951 \\
\hline No $(\%)$ & $16(72.7)$ & $9(69.2)$ & \\
\hline Yes $(\%)$ & $3(13.6)$ & $3(23.1)$ & \\
\hline Unknown (\%) & $3(13.6)$ & $1(7.7)$ & \\
\hline Protease inhibitor treatment & & & 0.251 \\
\hline Boceprevir (\%) & $9(40.9)$ & $8(61.5)$ & \\
\hline Telaprevir (\%) & $13(59.1)$ & $5(34.5)$ & \\
\hline
\end{tabular}

Table 3 shows treatment outcomes stratified by race: African-Americans to White Americans. The only significantly disparate variables related to BMI $(p=0.046)$ and initial ribavirin dose $(p=0.024)$. African-Americans had higher BMI at treatment initiation compared to White Americans (33.6 and 28.8, respectively), resulting in higher mean starting ribavirin doses in African-Americans (1,092 and $900 \mathrm{mg}$, respectively). There was no significant effect of race on cytopenia that required erythropoietin injection $(p=0.831)$, neupogen injection $(p=0.891)$, or blood transfusion $(p=$ 0.282 ) noted between the two groups. Additionally, there was not a significant trend $(p=0.528)$ in SVR12 rates between White Americans (81.0\%) and African-Americans (77\%).
Finally, there was no significant difference between races of pegylated interferon dosing at treatment initiation ( $p=$ $0.206)$ or lowest reduced dose $(p=0.213)$.

Table 4 compares the outcomes-based protease inhibitor used, boceprevir or telaprevir. Only viral negativity at week 4 $(p=0.000)$ differed between the two groups $-29 \%$ of the patients achieve rapid viral response (RVR) on boceprevir, while $89 \%$ of patients achieved RVR on telaprevir. This reflects the use of the "lead" in phase with boceprevir. However, SVR12 was ultimately achieved $79.4 \%$ of the time in both groups and was not significant $(p=1.00)$. Finally, there was no significant difference in cytopenia and blood product transfusion requirements between the two groups. 
Table 2 Treatment outcomes by SVR12

${ }^{\text {a }}$ SVR12 unknown for one of the cases as patient was lost to followup. SVR12 was defined as undetectable HCV RNA level at least 12 weeks after completion of treatment duration, which varies according to protease inhibitor, presence of cirrhosis, and dose modifications as per management decisions of hepatologist

${ }^{\mathrm{b}}$ Cirrhosis was determined according to radiographic, histologic, or serologic criteria

${ }^{c}$ Hospitalization includes ER visits or inpatient admission

${ }^{\mathrm{d}}$ Not all participants had known IL28B

\begin{tabular}{|c|c|c|c|}
\hline & No $\operatorname{SVR}^{\mathrm{a}}(n=7)$ & $\operatorname{SVR}^{\mathrm{a}}(n=27)$ & Significance \\
\hline BMI (SD) & $28.7(5.9)$ & $31(7.3)$ & 0.471 \\
\hline Lowest RBV dose in mg (SD) & $667(372)$ & $785(357)$ & 0.467 \\
\hline Lowest PEG dose in mcg (SD) & $147.5(38)$ & $155(41)$ & 0.698 \\
\hline Starting RBV dose in mg (SD) & $866.7(301)$ & $1,000(237)$ & 0.241 \\
\hline Starting PEG dose in mcg (SD) & $155(40)$ & $169(27)$ & 0.292 \\
\hline $\mathrm{RBV}$ reduced to $<600 \mathrm{mg}$ daily & $57 \%$ & $43 \%$ & 0.421 \\
\hline Age in years (SD) & $48(8.9)$ & $49.7(12.0)$ & 0.749 \\
\hline Married & $83 \%$ & $46 \%$ & 0.106 \\
\hline Needed RBV reduction & $67 \%$ & $46 \%$ & 0.384 \\
\hline Needed PEG reduction & $33 \%$ & $21 \%$ & 0.547 \\
\hline Job & $50 \%$ & $64 \%$ & 0.528 \\
\hline $\begin{array}{l}\text { Men } \\
\text { Women }\end{array}$ & $\begin{array}{l}22 \% \\
13 \%\end{array}$ & $\begin{array}{l}78 \% \\
88 \%\end{array}$ & 0.473 \\
\hline $\begin{array}{l}\text { White American }(n=22) \\
\text { African-American }(n=13)\end{array}$ & $\begin{array}{l}19 \% \\
23 \%\end{array}$ & $\begin{array}{l}81 \% \\
77 \%\end{array}$ & 0.528 \\
\hline Cirrhosis $^{\mathrm{b}}$ & $50 \%$ & $13 \%$ & 0.013 \\
\hline Needed epogen injection & $33 \%$ & $25 \%$ & 0.686 \\
\hline Needed neupogen & $0 \%$ & $18 \%$ & 0.276 \\
\hline Needed blood transfusion & $33 \%$ & $0 \%$ & 0.001 \\
\hline Hospitalized $^{\mathrm{c}}$ & $33 \%$ & $25 \%$ & 0.686 \\
\hline $\begin{array}{l}\text { IL28B genotype }^{\mathrm{d}}(\mathrm{CC})(n=9) \\
\text { IL28B genotype (CT) }(n=14)\end{array}$ & $\begin{array}{l}0 \% \\
14 \%\end{array}$ & $\begin{array}{l}100 \% \\
86 \%\end{array}$ & 0.007 \\
\hline IL28B genotype (TT) $(n=5)$ & $60 \%$ & $40 \%$ & \\
\hline $\begin{array}{l}\text { Tx naïve }(n=19) \\
\text { Prev nonresponser }(n=5)\end{array}$ & $\begin{array}{l}11 \% \\
40 \%\end{array}$ & $\begin{array}{l}89 \% \\
60 \%\end{array}$ & 0.589 \\
\hline Prev relapser $(n=8)$ & $25 \%$ & $75 \%$ & \\
\hline $\begin{array}{l}\text { Boceprevir } \\
\text { Telaprevir }\end{array}$ & $\begin{array}{l}18 \% \\
18 \%\end{array}$ & $\begin{array}{l}82 \% \\
82 \%\end{array}$ & 1.000 \\
\hline
\end{tabular}

\section{Discussion}

Contrary to results from several large, sponsored trials, this study of real-world outcomes with HCV triple therapy failed to confirm the SVR disparity between African-American and White-American patients seen in previous PEG/RBV-based treatment trials. SVR 12 with triple therapy was $81 \%$ in White Americans vs $77 \%$ in African-Americans $(p=0.528)$. In addition, real-world overall SVR rates were higher than observed in index trials by Poordad et al. [7] and Jacobson et al. [8]. Patients with IL28B polymorphism subtype CT/TT or cirrhosis were less likely to achieve SVR12, but this was regardless of race. Patients with severe anemia, requiring blood transfusions, were also less likely to achieve SVR12; however, this did not negatively alter treatment efficacy when stratified by race.

Prior to triple therapy, previous treatment regimens with PEG/RBV demonstrated a significant difference in SVR in African-Americans compared to non-African-Americans [16-19]. Despite the overall increase in SVR with first generation protease inhibitors, the SVR gap between White and African-Americans did not appear to be completely resolved.
In the index trial (SPRINT-2) for triple therapy using boceprevir by Poordad et al. (SPRINT-2), the SVR (modified ITT population) of group 2 (boceprevir + PEG/RBV for 24 weeks) and group 3 (boceprevir + PEG/RBV for 44 weeks) combined were $50 \%$ for African Americans and $70.4 \%$ for White Americans [7]. The odds ratio of SVR between black and non-Black cohorts was $0.5(p<0.001)$. Similarly, a difference was also seen in the index trial of telaprevir (ADVANCE-T12PR arm) by Jacobson et al [8]; SVR was achieved in $62 \%$ of African-Americans and $75 \%$ of White Americans. In our study, overall SVR was higher than published in the index trials, but SVR did not differ significantly between our African-American (77 \%) and White American $(81 \%)(p=0.53)$ cohorts. As seen in McCombs et al.'s recent report on Veterans Affairs' experience where overall SVR with telaprevir-based therapy where only $4 \%$ of treated subjects achieved a negative viral load, real-world success can often lag behind that seen in strictly conducted clinical trials [20]. It is reassuring to find these expensive medications can yield very favorable results in a nonstudy setting even in historically difficult to treat populations. 
Table 3 Treatment outcomes by race

\begin{tabular}{|c|c|c|c|}
\hline & White American $(n=22)$ & African-American $(n=13)$ & Significance \\
\hline BMI (SD) & $28.8(5.2)$ & $33.6(8.6)$ & 0.046 \\
\hline $\mathrm{VN}$ week $4^{\mathrm{a}}$ & $68 \%$ & $46 \%$ & 0.21 \\
\hline VN week 8 & $95 \%$ & $85 \%$ & 0.303 \\
\hline VN week 12 & $91 \%$ & $85 \%$ & 0.585 \\
\hline Starting mean RBV dose in mg (SD) & $900(244)$ & $1,092(210)$ & 0.024 \\
\hline Starting mean PEG dose in mcg (SD) & $162(34)$ & $175(16)$ & 0.206 \\
\hline Lowest mean RBV dose in mg (SD) & $709(347)$ & $800(424)$ & 0.496 \\
\hline Lowest mean PEG dose in mcg (SD) & $147(43)$ & $165(30)$ & 0.219 \\
\hline RBV dose $<600$ mg daily (\%) & 45 & 46 & 0.969 \\
\hline Required blood transfusion (\%) & $1(4.5)$ & $2(15.3)$ & 0.282 \\
\hline Required neupogen (\%) & $3(13.6)$ & $2(15.3)$ & 0.891 \\
\hline Required epogen $(\%)$ & $6(27.2)$ & $4(30.8)$ & 0.831 \\
\hline Protease inhibitor held ${ }^{\mathrm{b}}(\%)$ & $1(4.5)$ & $3(23.1)$ & 0.101 \\
\hline ANC decrease week 0 to 24 (SD) & $2,210(1395)$ & $2,820(2081)$ & 0.328 \\
\hline Hgb decrease (SD) & $3.37(1.4)$ & $2.38(2.0)$ & 0.114 \\
\hline Plt decrease $100 \mathrm{~K}$ (SD) & $62.4(52.8)$ & $71.3(49.0)$ & 0.187 \\
\hline $\operatorname{SVR} 12^{\mathrm{c}}(\%)$ & $17(80.9)$ & $10(76.9)$ & 0.528 \\
\hline
\end{tabular}

${ }^{\mathrm{a}} \mathrm{VN}-\mathrm{HCV}$ RNA viral level was undetectable at weeks 4, 8, and 12

${ }^{\mathrm{b}}$ Protease inhibitor held due to side effects with continuation of PEG/RBV or discontinuation of all medications

${ }^{\mathrm{c}}$ SVR12 unknown for one of the cases as patient was lost to follow-up. SVR12 was defined as undetectable HCV RNA level at least 12 weeks after completion of treatment duration, which varies according to protease inhibitor, presence of cirrhosis, and dose modifications as per management decisions of hepatologist

Table 4 Treatment outcomes by protease inhibitor

\begin{tabular}{llll}
\hline & Bocep $(n=17)$ & Tela $(n=18)$ & Significance \\
\hline BMI & $31.2(8.2)$ & $30.1(5.8)$ & 0.655 \\
VN week 4 & $29 \%$ & $89 \%$ & 0.000 \\
VN week 8 & $88 \%$ & $94 \%$ & 0.491 \\
EVR-week 12 & $82 \%$ & $94 \%$ & 0.274 \\
Lowest RBV dose in mg (SD) & $718(367)$ & $767(390)$ & 0.705 \\
Lowest PEG dose in mcg (SD) & $145(46)$ & $163(31)$ & 0.205 \\
RBV dose $<600$ mg daily (\%) & 41 & 11 & 0.613 \\
Required blood transfusion (\%) & 6 & 11 & 0.594 \\
Required neupogen (\%) & 18 & 28 & 0.594 \\
Required epogen (\%) & 29 & 17 & 0.918 \\
PI held (\%) & 6 & $2154(1325)$ & 0.331 \\
ANC decrease week 0 to 24 (SD) & $2723(1974)$ & $3.1(1.6)$ & 0.346 \\
Hgb decrease (SD) & $2.9(1.8)$ & $71.2(48.9)$ & 0.731 \\
Plt decrease 100 K (SD) & $6.8(54.2)$ & $14(82)^{\mathrm{d}}$ & 0.609 \\
SVR12 (\%) & $14(82)$ & & 1.000
\end{tabular}

${ }^{a} \mathrm{VN}-\mathrm{HCV}$ RNA viral level was undetectable at weeks 4 and 8

${ }^{\mathrm{b}}$ Protease inhibitor held due to side effects with continuation of PEG/RBV or discontinuation of all medications

${ }^{\mathrm{c}}$ SVR12 unknown for one of the cases as patient was lost to follow-up. SVR12 was defined as undetectable HCV RNA level at least 12 weeks after completion of treatment duration, which varies according to protease inhibitor, presence of cirrhosis, and dose modifications as per management decisions of hepatologist

${ }^{\mathrm{d}}$ Unknown SVR12 in one patient 
Several characteristics of our cohort, such as baseline HCV RNA, genotype, and fibrosis score may help to explain the differences seen in overall SVR between our study and the index trials for boceprevir (groups 2 and 3) and telaprevir (T12PR)-See Supplemental Table 1. Baseline HCV RNA $>800,000$ were higher in the boceprevir trial $(85 \%$ of nonBlacks, $91 \%$ of Blacks) as well as the telaprevir trial (overall $77 \%$ ). In our study, $69 \%$ of African-Americans and $64 \%$ of White Americans had a baseline HCV RNA $>800,000$. Also, patients with genotype 1a tend to be more responsive to PEG/ RBV dual therapy [21]. Our study had slightly higher percentage of subtype 1a (76.9-African-American, $91 \%$ White American) compared to the boceprevir trial (75\%Black, $65 \%$-non-Black) and the telaprevir trial (59\% overall). An enrichment of genotype 1a patients may partially explain the high SVR rate we observed.

We feel our findings have external validity given that we were able to confirm several factors reported in the large sponsored trials. For example, the distribution of IL-28B genotypes was consistent with previous trials as was the relative influence of advanced liver fibrosis on SVR rates. In telaprevir trial (ADVANCE-T12PR arm), $20 \%$ of included subjects were Metavir fibrosis stage 3 or 4 [8]. Patients with cirrhosis were 2.5 times less likely to achieve SVR in that trial. In the boceprevir trial (SPRINT-2), as expected, SVR rates were also higher in patients without cirrhosis (i.e., Metavir stage 0,1 , or 2 ). Based on clinical judgment regarding radiographic, serologic, and pathologic evidence, approximately $15 \%$ of African-Americans and $14 \%$ of White Americans had cirrhosis in our cohort. As with these original studies, cirrhosis was a significant predictor of treatment failure in our real-world data as well, where only $40 \%$ of cirrhotic patients obtained SVR12 compared to $88 \%$ of patients without evidence of advanced fibrosis obtained SVR12.

It remains unclear as to why patients with severe anemia who received blood transfusions were less likely to achieve SVR12 in our study. Although there were differences in BMI and subsequently RBV dosing between the AA and the non-AA study groups, SVR12 was not statistically significant between these two groups. Erythropoietin injections and blood transfusions have been used historically in order to allow patients to maintain higher levels of RBV and in turn, optimize their chances at SVR. No randomized studies have evaluated erythropoietin use in achieving SVR. Similarly, no conclusive data exists about transfusion requirements predicting treatment outcomes. Recently, Poordad et al. [22] evaluated the management of anemia in patients on triple therapy (boceprevir) and concluded that RBV reduction was an effective primary management approach. Additionally, RBV dose reduction did not affect SVR. Our observation of a negative correlation between transfusion and SVR does nothing to refute Poordad et al.'s findings.
Consideration must also be given to the mechanisms of action of the individual medications comprising the regimens, and how newer, direct-acting anti-viral agents have improved outcomes. As the previous standard of care, IFN/RBV therapy had less than $50 \%$ efficacy in the treatment of chronic HCV. Pegylated-interferon is an immunoregulatory cytokine with profound effects on immune system responses to viral antigens. This activation is not virus-specific, and genetic variants (both host and pathogen) allow for resistance and varied responses to treatment. Consensus opinion has suggested that the innate and adaptive immune responses, host genetic variables, genetic diversity of the virus, and clinical comorbidities (diabetes) may all account for the ineffectiveness of IFN/RBV in the treatment of HCV [23].

First generation direct-acting antiviral agents (DAA), specifically boceprevir and telaprevir, are specific inhibitors of viral proteases, leading to direct inhibition of the processing of viral proteins critical to viral replication. Although the addition of first-generation DAA's was a significant improvement to existing IFN/RBV regimens, there were still differences in treatment outcomes based upon race, genotype, IL28B polymorphism, and severity of fibrosis. The next generation of DAAs (combinations of sofosbuvir and ledipasvir, which inhibit multiple viral targets simultaneously) [24, 25] have demonstrated superior SVR12 rates as well as equivalent efficacy in challenging populations such as African-Americans, non-CC IL28B polymorphism, and cirrhotics. These results suggest that combination regimens of direct-acting antivirals may transcend patient factors (race, IL28B polymorphism, and genotype) that have previously limited treatment options for these more challenging cohorts.

There are several limitations inherent to this retrospective evaluation. Chiefly, there may be an element of selection bias in which patients to treat. While AfricanAmericans have historically been less likely to be eligible for pharmaceutical studies compared to White Americans [4], it is possible that this group of nontrial patients was disproportionately represented in this study. This may yield patients with more complicated medical and socioeconomic cofactors that both positively or negatively influence outcomes. Thirdly, the strategies used for anemia-blood transfusions, erythropoietin injection, or ribavirin reduction-may not be consistent with recommended treatment protocols in study trials. This could place more emphasis on blood transfusion as an indicator of treatment outcome. Finally, as this was a retrospective analysis, there was some missing data (labs, biopsy) and inconsistent follow-up visits that could not be accounted for our secondary endpoint analyses, and could possibly influence these results 


\section{Conclusion}

We studied the treatment outcomes of 35 consecutive nonstudy patients who represented a balanced panel based upon disease prevalence and racial demographics. Many risk factors for treatment outcome, such as IL28B genotype and presence of cirrhosis, were consistent with the index trials SPRINT-2 and ADVANCE. However, we found SVR may not be lower in AA as compared to non-AA treated with first generation protease inhibitors in combination with pegylated interferon and ribavirin. Real-world SVR rates may actually be consistent with or higher than the index trial results. Finally, the treatment of severe anemia with red cell transfusion is negatively associated with SVR.

Acknowledgments The authors would like to thank Ward Miller, Stephany Luke, Stephanie Walker, and Michelle Barbarin.

Conflict of Interest This research was not sponsored by any organization or company. The design and execution of this observational study was prompted by clinical curiosity alone, without any support from the companies that make these medications. John Wysocki and Celeste Newby declare that they have no conflict of interest. Luis Balart is on advisory committees or review panels for Genentech, Janssen, AbbVie; received grant/research support from Merck, Roche/Genentech, Bayer, Hyperion, AbbVie, Takeda, GI Dynamics, Gilead, BMS, Eisai, Vertex; and does speaking and teaching for Merck. Nathan Shores does speaking and teaching for Gilead.

Disclosure No animal or human studies were carried out by the authors for this article.

\section{References}

1. Pyrsopoulos N, Jeffers L. Hepatitis C in African Americans. J Clin Gastroenterol. 2007;41(2):185-93.

2. Seeff LB et al. 45-year follow-up of hepatitis $C$ virus infection in healthy young adults. Ann Intern Med. 2000;132(2):105-11.

3. Alter MJ et al. The prevalence of hepatitis $\mathrm{C}$ virus infection in the United States, 1988 through 1994. N Engl J Med. 1999;341(8):55662.

4. Melia MT et al. Racial differences in hepatitis $\mathrm{C}$ treatment eligibility. Hepatology. 2011;54(1):70-8.

5. Davis GL et al. Aging of hepatitis $\mathrm{C}$ virus (HCV)-infected persons in the United States: a multiple cohort model of HCV prevalence and disease progression. Gastroenterology. 2010;138(2):513-21. 521.e1-6.

6. Ly KN et al. The increasing burden of mortality from viral hepatitis in the United States between 1999 and 2007. Ann Intern Med. 2012;156(4):271-8.
7. Poordad $\mathrm{F}$ et al. Boceprevir for untreated chronic HCV genotype 1 infection. N Engl J Med. 2011;364(13):1195-206.

8. Jacobson IM et al. Telaprevir for previously untreated chronic hepatitis C virus infection. N Engl J Med. 2011;364(25):2405-16.

9. Bacon BR et al. Boceprevir for previously treated chronic HCV genotype 1 infection. N Engl J Med. 2011;364(13):1207-17.

10. Sherman KE et al. Response-guided telaprevir combination treatment for hepatitis $\mathrm{C}$ virus infection. $\mathrm{N}$ Engl J Med. 2011;365(11):1014-24.

11. Hezode $\mathrm{C}$ et al. Telaprevir and peginterferon with or without ribavirin for chronic HCV infection. N Engl J Med. 2009;360(18):1839-50.

12. McHutchison JG et al. Telaprevir with peginterferon and ribavirin for chronic HCV genotype 1 infection. N Engl J Med. 2009;360(18): 1827-38.

13. McHutchison JG et al. Telaprevir for previously treated chronic HCV infection. N Engl J Med. 2010;362(14):1292-303.

14. Kwo PY et al. Efficacy of boceprevir, an NS3 protease inhibitor, in combination with peginterferon alfa- $2 \mathrm{~b}$ and ribavirin in treatmentnaive patients with genotype 1 hepatitis $\mathrm{C}$ infection (SPRINT-1): an open-label, randomised, multicentre phase 2 trial. Lancet. 2010;376(9742):705-16.

15. Chen $\mathrm{J}$ et al. Earlier sustained virologic response end points for regulatory approval and dose selection of hepatitis $\mathrm{C}$ therapies. Gastroenterology. 2013;144(7):1450-1455.e2.

16. Muir AJ, Bornstein JD, Killenberg PG. Peginterferon alfa-2b and ribavirin for the treatment of chronic hepatitis $\mathrm{C}$ in blacks and nonHispanic whites. N Engl J Med. 2004;350(22):2265-71.

17. Conjeevaram HS et al. Peginterferon and ribavirin treatment in African American and Caucasian American patients with hepatitis C genotype 1. Gastroenterology. 2006;131(2):470-7.

18. Reddy KR et al. Racial differences in responses to therapy with interferon in chronic hepatitis C. Consensus Interferon Study Group. Hepatology. 1999;30(3):787-93.

19. Jeffers LJ et al. Peginterferon alfa- $2 \mathrm{a}(40 \mathrm{kd})$ and ribavirin for black American patients with chronic HCV genotype 1. Hepatology. 2004;39(6):1702-8

20. McCombs $\mathrm{J}$ et al. The risk of long-term morbidity and mortality in patients with chronic hepatitis $\mathrm{C}$ : results from an analysis of data from a Department of Veterans Affairs Clinical Registry. JAMA Intern Med. 2013;174(2):204-12.

21. Pellicelli AM et al. HCV genotype 1a shows a better virological response to antiviral therapy than $\mathrm{HCV}$ genotype $1 \mathrm{~b}$. BMC Gastroenterol. 2012;12:162.

22. Poordad $\mathrm{F}$ et al. Effects of ribavirin dose reduction vs erythropoietin for boceprevir-related anemia in patients with chronic hepatitis $\mathrm{C}$ virus genotype 1 infection - a randomized trial. Gastroenterology. 2013;145(5):1035-1044.e5.

23. Chung RT, Gale Jr M, Polyak SJ, Lemon SM, Liang TJ, Hoofnagle $\mathrm{JH}$. Mechanisms of Action of interferon and ribavirin in chronic hepatitis C: summary of a workshop. Hepatology. 2008;47(1):30620.

24. Afdhal N, Zeuzem S, Kwo P, Chojkier M, Gitlin N, Puoti M, et al. Ledipasvir and sofosbuvir for untreated HCV genotype 1 infection. N Engl J Med. 2014;370(20):1889-98.

25. Afdhal N, Reddy KR, Nelson DR, Lawitz E, Gordon SC, Schiff E, et al. Ledipasvir and sofosbuvir for previously treated HCV genotype 1 infection. N Engl J Med. 2014;370(16):1483-93. 